\title{
A computational approach for shallow water forced Korteweg-De Vries equation on critical flow over a hole with three fractional operators
}

\author{
Pundikala Veeresha $^{a}$, Mehmet Yavuz $^{b, c^{*}}$ and Chandrali Baishya ${ }^{d}$ \\ ${ }^{a}$ Center for Mathematical Needs, Department of Mathematics, CHRIST (Deemed to be University), \\ Bengaluru-560029, India \\ ${ }^{b}$ Department of Mathematics and Computer Sciences, Faculty of Science, Necmettin Erbakan University, \\ 42090 Konya, Turkey \\ ${ }^{c}$ Department of Mathematics, College of Engineering, Mathematics and Physical Sciences, University of \\ Exeter, TR10, Cornwall, UK \\ ${ }^{d}$ Department of Studies and Research in Mathematics, Tumkur University, Tumkur-572103, India \\ pundikala.veeresha@christuniversity.in, M.Yavuz@exeter.ac.uk, baishyachandrali@gmail.com
}

\section{ARTICLE INFO}

\section{Article History:}

Received 12 October 2020

Accepted 9 December 2021

Available 31 December 2021

Keywords:

Force KdV equation

Fractional derivatives

q-Homotopy analysis transform

technique

Fixed point theorem

AMS Classification 2010:

26A33; 65M99; 35J05

\section{ABSTRACT}

The Korteweg-De Vries (KdV) equation has always provided a venue to study and generalizes diverse physical phenomena. The pivotal aim of the study is to analyze the behaviors of forced KdV equation describing the free surface critical flow over a hole by finding the solution with the help of $q$-homotopy analysis transform technique ( $q$-HATT). he projected method is elegant amalgamations of $q$-homotopy analysis scheme and Laplace transform. Three fractional operators are hired in the present study to show their essence in generalizing the models associated with power-law distribution, kernel singular, non-local and non-singular. The fixed-point theorem employed to present the existence and uniqueness for the hired arbitrary-order model and convergence for the solution is derived with Banach space. The projected scheme springs the series solution rapidly towards convergence and it can guarantee the convergence associated with the homotopy parameter. Moreover, for diverse fractional order the physical nature have been captured in plots. The achieved consequences illuminates, the hired solution procedure is reliable and highly methodical in investigating the behaviours of the nonlinear models of both integer and fractional order.

(cc) $\mathrm{BY}$

\section{Introduction}

Mankind is always looking for innovation, development, novelty, modernization and modification in science and technology to lead daily life in a convenient manner. In this connection, mathematics is the basic, essential and pivotal tool and which aid us to study, investigate and predict the essence of life associated with surrounding nature. Among this tool, calculus with differential and integral operators is the most efficient and favourable instrument and it has been recanalized most elegant discipline. Most of the concept in nature associated with the rate of change, variation and modification are necessitates differential calculus. Recently, many researchers came with limitations of classical concept particularly to capture power-law, non-local, non-singular, heterogeneities, exponential decay, fading memory, fatigue effect, and other functions. Later, mathematicians suggested an old tool and which is rooted soon after the classical concept, called fractional calculus (FC). Many senior pioneers prearranged the reputation of $\mathrm{FC}$ and proposed distinct

*Corresponding Author 
notions and properties $1-15]$. In a while, the fundamental theory and extensive claims of essential properties are broadly employed to model diverse physical mechanisms and everyday problems 1622 .

The essence of studying the mathematical models with differential equations of fractional and integer order is always a hot topic, and hence many researchers are magnetized towards the new approaches with numerical and analytical methods. For instance, authors in 23 find the invariant solution for Bogoyavlensky-Konopelchenko equation, the fractional-reaction diffusion trimolecular models is studied in [24], the fractional-order Gross-Pitaevskii equations are examined with the help of unified method by researchers in 25. Similarly, authors derived interesting results for Calogero-Bogoyavlenskii-Schif 26 and coupled Korteweg-de Vries equations [27] with similar techniques. To show the essence of the Lie symmetry analysis, authors in 28 investigated about the Bratu Gelfand model, the effect of fractional derivatives are illustrated by authors to capture the stimulating results associated with fifth-order Schrodinger equation [29], COVID-19 [30, Maccari systems [31], chaotic system [32], the mathematical model of Tumour invasion and metastasis [33, and modified coupled Korteweg-de Vries system [34]. The Lump and optical solitons solutions are derived by researchers in [35] with the analytical method, and authors in $[36 \mid$ derived some stimulating results associated with bipartite graph and fractional operator. The projected method is hired by the scholars to investigate the system associated with Jaulent-Miodek system with energy-dependent Schrödinger potential [37], the epidemic model of childhood disease [38], liquids with gas bubbles models [39], the Zakharov-Kuznetsov equation in dusty plasma [40], and Degasperis-Procesi equations 41 .

In a two-dimensional channel flow, the impact of bottom configurations on the free-surface waves is investigated with the help of the forced Kortewegde Vries equation. The bottom topography plays a vital part in the study of shallow-water waves, and which can significantly evaluate the behaviours of wave motions [42,43]. Shallow water or long waves are the waves in water shallower than 1/20 their actual wavelength. When the bottom configuration is more complex, the interplay between the bottom topography and solitary waves can demonstrate more stimulating dynamics of the free surface waves. When the rigid bottom of the channel has some obstacles and for an incompressible and inviscid fluid, the twodimensional channel flow with free surface waves have been studied [44, 45]. Fluid flows over an obstacle, the forcing approximately with the $\mathrm{KdV}$ equation can portray the development of the free surface. The FKdV equation is very important while describing the nature sine Gordon equation as well as the nonlinear Schrödinger equation. Further, the proposed model has numerous applications in the connected branches of mathematics and physics. This equation is considered an essential tool to study the propagation of short laser pulses in optical fibres, atmosphere dynamics, geostrophic turbulence and magnetohydrodynamic waves 46, 47. Particularly, it offers stimulating results associated to physical problems such as acoustic waves on a crystal lattice, tsunami waves over obstacles, and shallow-water waves over rocks.

Here, we consider the forced $\mathrm{KdV}$ equation with the free water surface elevation measured $u(x, t)$ on critical flow over a hole from undisturbed water level and which introduce and nurtured by $\mathrm{Wu}$ in 1987 [48], and presented as follows:

$$
\begin{array}{r}
\frac{1}{c} \frac{\partial u}{\partial t}+\left[\left(F_{r}-1\right)-\frac{3}{2} \frac{u(x, t)}{h}\right] \frac{\partial u}{\partial x} \\
-\frac{h^{2}}{6} \frac{\partial^{3} u}{\partial x^{3}}=\frac{1}{2} \frac{\partial f(x)}{\partial x},
\end{array}
$$

where $F_{r}$ is Froude number and it also calls as the critical parameter, $h$ is the sea mean water depth, $f(x)$ is the external forcing term and define as $f(x)=\frac{p_{a}(x)}{\rho g}+b(x)$. Here, $\frac{p_{a}(x)}{\rho g}$ is the surface air pressure, and $b(x)$ is rigid bottom topography and is defined by $b(x)=-0.1 e^{-\frac{x^{\beta}}{4}}-1$. The Froude number $\left(F_{r}\right)$ plays an important role in Eq. (1), since its value elucidates the kind of critical flows over the localised obstacle. Specifically, for $>1,=1$ and $<1$ respectively represent the flow is considered as supercritical, trans-critical and subcritical. In the rigid bottom topography $b(x)$, two different kinds of hole examined, namely for $\beta=2$ and $\beta=8$. The behaviours of $b(x)$ for two distinct cases is cited in Figure 1. These cases respectively signify the hole is expected an inverse of bell-shaped and the hole is more flattened at the bottom as well as wider. Authors in [49], considered the simplified above equation by eliminating surface air pressure and presented it as follows

$$
\begin{array}{r}
\frac{1}{c} \frac{\partial u}{\partial t}+\left[\left(F_{r}-1\right)-\frac{3}{2} \frac{u(x, t)}{h}\right] \frac{\partial u}{\partial x} \\
-\frac{h^{2}}{6} \frac{\partial^{3} u}{\partial x^{3}}-\frac{1}{2} \frac{\partial b(x)}{\partial x}=0 .
\end{array}
$$


In the literature, we have diverse fractional operators and the most familiarly used are including Riemann-Liouville (RL), Caputo [3], CaputoFabrizio (CF) [50] and Atangana-Baleanu (AB) [51] operators. However, mathematicians and scientists are always looking and searching for the tool which can help to derive and find the required consequences at a particular situation in specific context. In this regard, each earlier proposed concepts have their own confines. Including, the RL operator be unsuccessful to admit the universal truth of derivative and then M. Caputo suggested new notion which overcame this drawback. Recently, researchers cited some additional properties need to be incorporate with this operator and many new fractional operators with their own merits are suggested by mathematicians.

Recently, many researchers are hired them as generalizing tool to investigate diverse phenomena and achieved some stimulating consequences [6, 16, 43]. Particularly, these operators aid us to investigate the long-range memory, heterogeneities, exponential decay and non-local and non-singular, non-Gaussian without a steadystate and crossover behaviour. Now, we consider the fractional-order forced $\mathrm{KdV}$ (FF-KdV) equation by trading the time derivative with three fractional operators. Now, the FF-KdV equation is defined as follows

$$
\begin{aligned}
D_{t}^{\alpha} u(x, t)= & -c\left(\left[\left(F_{r}-1\right)-\frac{3}{2} \frac{u(x, t)}{h}\right] \frac{\partial u}{\partial x}\right. \\
& \left.-\frac{h^{2}}{6} \frac{\partial^{3} u}{\partial x^{3}}-\frac{1}{2} \frac{\partial b(x)}{\partial x}\right), \\
{ }_{0}^{C F} D_{t}^{\alpha} u(x, t) & =-c\left(\left[\left(F_{r}-1\right)-\frac{3}{2} \frac{u(x, t)}{h}\right] \frac{\partial u}{\partial x}\right. \\
& \left.-\frac{h^{2}}{6} \frac{\partial^{3} u}{\partial x^{3}}-\frac{1}{2} \frac{\partial b(x)}{\partial x}\right), \\
{ }_{a}^{A B C} D_{t}^{\alpha} u(x, t) & =-c\left(\left[\left(F_{r}-1\right)-\frac{3}{2} \frac{u(x, t)}{h}\right] \frac{\partial u}{\partial x}\right. \\
& \left.-\frac{h^{2}}{6} \frac{\partial^{3} u}{\partial x^{3}}-\frac{1}{2} \frac{\partial b(x)}{\partial x}\right),
\end{aligned}
$$

where $\alpha(0<\alpha \leq 1)$ is fractional-order. The considered model offers an interesting insight into diverse physical phenomena and hence it magnetizes researchers with different tools to present their viewpoints with corresponding derived consequences. For instance, authors in model 52 find the analytic solutions to the projected model; author in [53] presents some interesting result for the proposed model; considering the model for waves generated by topography, authors in 49,54 find the approximated analytical solution by using the HAM; authors in [55] investigated the considered problem and presented dynamics of trapped solitary waves; lines and pseudospectral solutions has been investigated by authors in [56].

The hired scheme is a blend of Laplace transform (LT) with homotopy based scheme [57, 58]. The uniqueness of $q$-HATT is that it does not require assumptions, perturbations, conversion of nonlinear to linear and PDE to ODE [59]. Moreover, it is the generalization of many methods (results attained by this technique is a particular case for the value of parameters associated to method). The projected algorithm has been employed due to its efficiency and accuracy to examine the extensive classes of complex as well as nonlinear models and problems and also for the system of equations 60 67]. Recently, many interesting consequences are derived by using the projected scheme while analyzing the real-world problem.

The rest of the manuscript is systematized as follows: We followed the next section by basics and essential notions of both FC and LT. In Section 3, the solution for the hired model with three fractional operators are presented and also the existence and uniqueness of solutions with two fractional operators for the model is presented using Banach space within the frame of fixed-point theory. With the aid of attained outcomes and corresponding consequences, the discussion about the results is presented in Section 4 and finally, the concluding remarks on the present study are presented in the lost segment.

\section{Preliminaries}

Here, we recall few basic notions of FC $3,50,51$, 68, 69]:

Definition 1. The Caputo fractional derivative of $f \in C_{-1}^{n}$ is presented for $n \in \mathbb{N}$ as

$$
\begin{aligned}
& D_{t}^{\alpha} f(t)=\frac{d^{n} f(t)}{d t^{n}}, \quad \alpha=n \in \mathbb{N}, \\
& =\frac{1}{\Gamma(n-\alpha)} \int_{0}^{t}(t-\vartheta)^{n-\alpha-1} f^{(n)}(\vartheta) d \vartheta, n-1<\alpha<n .
\end{aligned}
$$

Definition 2. The fractional Caputo-Fabrizio $(\mathrm{CF})$ derivative in Caputo sense for a function $f \in H^{1}(a, b)(b>a)$ is presented as follows 68

$$
\begin{aligned}
& { }_{0}^{C F C} D_{t}^{\alpha}(f(t)) \\
& =\frac{\mathcal{M}[\alpha]}{1-\alpha} \int_{0}^{t} f^{\prime}(\vartheta) \exp \left[-\frac{\alpha(t-\vartheta)}{1-\alpha}\right] d \vartheta,
\end{aligned}
$$

where $\mathcal{M}[\alpha](\mathcal{M}[0]=\mathcal{M}[1]=1)$ is a normalization function.

Definition 3. The fractional Atangana-BaleanuCaputo derivative for $f \in H^{1}(a, b)(b>a)$ is 


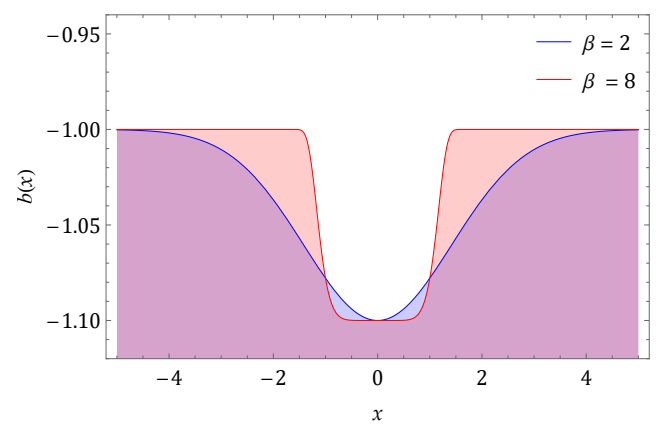

Figure 1. Nature of $b(x)$ at $\beta=2$ and 8 .

$$
\begin{aligned}
& { }_{a}^{A B C} D_{t}^{\alpha}(f(t)) \\
& =\frac{\mathcal{M}[\alpha]}{1-\alpha} \int_{a}^{t} f^{\prime}(\vartheta) E_{\alpha}\left[-\frac{\alpha(t-\vartheta)^{\alpha}}{1-\alpha}\right] d \vartheta .
\end{aligned}
$$

Definition 4. The fractional AB integral is presented as

$$
\begin{gathered}
{ }_{a}^{A B} I_{t}^{\alpha}(f(t))=\frac{1-\alpha}{\mathcal{M}[\alpha]} f(t) \\
+\frac{\alpha}{\mathcal{M}[\alpha] \Gamma(\alpha)} \int_{a}^{t} f(\vartheta)(t-\vartheta)^{\alpha-1} d \vartheta .
\end{gathered}
$$

Definition 5. The Laplace transform $(L T)$ for a Caputo fractional derivative $D_{t}^{\alpha} f(t)$ is defined for $(n-1<\alpha \leq n)$, as

$$
\mathcal{L}\left[D_{t}^{\alpha} f(t)\right]=s^{\alpha} F(s)-\sum_{r=0}^{n-1} s^{\alpha-r-1} f^{(r)}\left(0^{+}\right),
$$

where $F(s)$ is LT of $f(t)$.

Note: According to 68, the following must hold

$$
\frac{2(1-\alpha)}{(2-\alpha) \mathcal{M}(\alpha)}+\frac{2 \alpha}{(2-\alpha) \mathcal{M}(\alpha)}=1,0<\alpha<1
$$

which gives $\mathcal{M}(\alpha)=\frac{2}{2-\alpha}$. By the assist of the above equation researchers in 68 proposed a novel Caputo derivative for $0<\alpha<1$ as follows

$D_{t}^{\alpha}(f(t))=\frac{1}{1-\alpha} \int_{0}^{t} f^{\prime}(t) \exp \left[\alpha \frac{t-\vartheta}{1-\alpha}\right] d \vartheta$

Definition 6. The $L T$ for a $C F$ derivative ${ }_{0}^{C F C} D_{t}^{\alpha} f(t)$ is presented as below

$$
\begin{gathered}
\mathcal{L}\left[\begin{array}{ll}
{ }_{0} F C & D_{t}^{(\alpha+n)} f(t)
\end{array}\right] \\
=\frac{s^{n+1} \mathcal{L}[f(t)]-s^{n} f(0)-s^{n-1} f^{\prime}(0)-\cdots-f^{n}(0)}{s+(1-s) \alpha} .
\end{gathered}
$$

Definition 7. The LT of AB derivative is defined by

$$
\begin{aligned}
& \mathcal{L}\left[\begin{array}{l}
{ }_{0}^{A B R} \\
0
\end{array} D_{t}^{\alpha}(f(t))\right] \\
& =\frac{\mathcal{B}[\alpha]}{1-\alpha} \frac{s^{\alpha} \mathcal{L}[f(t)]-s^{\alpha-1} f(0)}{s^{\alpha}+(\alpha /(1-\alpha))} .
\end{aligned}
$$

Theorem 1. The Lipschitz conditions for the $R L$ and $A B$ derivatives are respectively held the following results [51]

$$
\begin{array}{r}
\left\|{ }_{a}^{A B R} D_{t}^{\alpha} f_{1}(t)-{ }_{a}^{A B R} D_{t}^{\alpha} f_{2}(t)\right\| \\
<K_{1}\left\|f_{1}(x)-f_{2}(x)\right\|,
\end{array}
$$

and

$$
\begin{aligned}
\left\|{ }_{a}^{A B C} D_{t}^{\alpha} f_{1}(t)-{ }_{a}^{A B C} D_{t}^{\alpha} f_{2}(t)\right\| \\
<K_{2}\left\|f_{1}(x)-f_{2}(x)\right\| .
\end{aligned}
$$

Theorem 2. The fractional-order differential equation ${ }_{a}^{A B C} D_{t}^{\alpha} f_{1}(t)=s(t)$ has a unique solution [51] and which is

$$
\begin{gathered}
f(t)=\frac{1-\alpha}{\mathcal{B}[\alpha]} s(t) \\
+\frac{\alpha}{\mathcal{B}[\alpha] \Gamma(\alpha)} \int_{0}^{t} s(\varsigma)(t-\varsigma)^{\alpha-1} d \varsigma .
\end{gathered}
$$

\section{Solution for FKDV equation}

The considered solution procedure is presented for the FKDV equation with three fractional operators to find the series solution. Further, for both $\mathrm{CF}$ and $\mathrm{AB}$ fractional operators existence and uniqueness is derived with fixed point theory.

\subsection{Caputo Sense}

Consider the equation defined in Eq. (3)

$$
\begin{gathered}
D_{t}^{\alpha} u(x, t)+c\left(\left[\left(F_{r}-1\right)-\frac{3}{2} \frac{u}{h}\right] \frac{\partial u}{\partial x}\right. \\
\left.-\frac{h^{2}}{6} \frac{\partial^{3} u}{\partial x^{3}}-\frac{1}{2} \frac{\partial b(x)}{\partial x}\right)=0,
\end{gathered}
$$

with

$$
u(x, 0)=-\frac{2 e^{x}}{\left(1+e^{x}\right)^{2}} .
$$

Taking $L T$ on Eq. (18) and using Eq. (19), we get 


$$
\begin{gathered}
\mathcal{L}[u(x, t)]=\frac{1}{s}\left(-\frac{2 e^{x}}{\left(1+e^{x}\right)^{2}}\right) \\
-\frac{c}{s^{\alpha}} \mathcal{L}\left\{\left[\left(F_{r}-1\right)-\frac{3}{2} \frac{u}{h}\right] \frac{\partial u}{\partial x}-\frac{h^{2}}{6} \frac{\partial^{3} u}{\partial x^{3}}-\frac{1}{2} \frac{\partial b(x)}{\partial x}\right\} .
\end{gathered}
$$

Now, $\mathcal{N}$ is presented as

$$
\begin{gathered}
\mathcal{N}[\varphi(x, t ; q)]=\mathcal{L}[\varphi(x, t ; q)]-\frac{1}{s}\left(-\frac{2 e^{x}}{\left(1+e^{x}\right)^{2}}\right) \\
+\frac{c}{s^{\alpha}} \mathcal{L}\left\{\left[\left(F_{r}-1\right)-\frac{3}{2} \frac{\varphi(x, t ; q)}{h}\right] \frac{\partial \varphi(x, t ; q)}{\partial x}\right. \\
\left.-\frac{h^{2}}{6} \frac{\partial^{3} \varphi(x, t ; q)}{\partial x^{3}}-\frac{1}{2} \frac{\partial b(x)}{\partial x}\right\} .
\end{gathered}
$$

At $\mathcal{H}(x, t)=1$, the deformation equation presented as

$\mathcal{L}\left[u_{m}(x, t)-k_{m} u_{m-1}(x, t)\right]=\hbar \Re_{m}\left[\vec{u}_{m-1}\right]$,

where

$$
\begin{aligned}
& \mathfrak{R}_{m}\left[\vec{u}_{m-1}\right]=\mathcal{L}\left[u_{m-1}(x, t)\right] \\
& -\left(1-\frac{k_{m}}{n}\right)\left\{\frac{1}{s}\left(-\frac{2 e^{x}}{\left(1+e^{x}\right)^{2}}\right)\right\} \\
& +\frac{c}{s^{\alpha}} \mathcal{L}\left\{\left(F_{r}-1\right) \frac{\partial u_{m-1}}{\partial x}-\frac{3}{2 h} \sum_{i=0}^{m-1} u_{i} \frac{\partial u_{m-1-i}}{\partial x}\right. \\
& \left.-\frac{h^{2}}{6} \frac{\partial^{3} u_{m-1}}{\partial x^{3}}-\frac{1}{2} \frac{\partial b(x)}{\partial x}\right\} .
\end{aligned}
$$

On employing inverse $L T$ on Eq. (22), one can get

$u_{m}(x, t)=k_{m} u_{m-1}(x, t)+\hbar \mathcal{L}^{-1}\left\{\Re_{m}\left[\vec{u}_{m-1}\right]\right\}$

On simplifying the above equations by assist of $u_{0}(x, t)=-\frac{2 e^{x}}{\left(1+e^{x}\right)^{2}}$ we can evaluate terms of series

$$
u(x, t)=u_{0}(x, t)+\sum_{m=1}^{\infty} u_{m}(x, t)\left(\frac{1}{n}\right)^{m} .
$$

as

$$
\begin{gathered}
u_{1}(x, t)=\frac{\hbar t^{\alpha}}{\Gamma[\alpha+1]}\left(c \left(\frac{6 \mathrm{e}^{2 x}\left(-1+\mathrm{e}^{x}\right)}{\left(1+\mathrm{e}^{x}\right)^{5} h}\right.\right. \\
-\frac{\mathrm{e}^{x}\left(-1+11 \mathrm{e}^{x}-11 \mathrm{e}^{2 x}+\mathrm{e}^{3 x}\right) h^{2}}{3\left(1+\mathrm{e}^{x}\right)^{5}}-0.025 \mathrm{e}^{-\frac{x^{2}}{4}} x \\
\left.\left.+\frac{2 \mathrm{e}^{x}\left(-1+\mathrm{e}^{x}\right)\left(-1+F_{r}\right)}{\left(1+\mathrm{e}^{x}\right)^{3}}\right)\right),
\end{gathered}
$$

\subsection{Caputo-Fabrizio Sense}

Consider the equation defined in Eq. (4)

$$
\begin{aligned}
& { }_{0}^{C F} D_{t}^{\alpha} u(x, t)+c\left(\left[\left(F_{r}-1\right)-\frac{3}{2} \frac{u}{h}\right]\right. \\
& \left.\frac{\partial u}{\partial x}-\frac{h^{2}}{6} \frac{\partial^{3} u}{\partial x^{3}}-\frac{1}{2} \frac{\partial b(x)}{\partial x}\right)=0,
\end{aligned}
$$

with initial conditions Eq. (19). Taking $L T$ on Eq. 26) and by the assist of Eq. (19), we get

$$
\begin{gathered}
\mathcal{L}[u(x, t)]=\frac{1}{s}\left(-\frac{2 e^{x}}{\left(1+e^{x}\right)^{2}}\right)-c \frac{s+(1-s) \alpha}{s} \\
\mathcal{L}\left\{\left[\left(F_{r}-1\right)-\frac{3}{2} \frac{u}{h}\right] \frac{\partial u}{\partial x}-\frac{h^{2}}{6} \frac{\partial^{3} u}{\partial x^{3}}-\frac{1}{2} \frac{\partial b(x)}{\partial x}\right\} .
\end{gathered}
$$

Now, $\mathcal{N}$ is defined as

$$
\begin{gathered}
\mathcal{N}[\varphi(x, t ; q)]=\mathcal{L}[\varphi(x, t ; q)]-\frac{1}{s}\left(-\frac{2 e^{x}}{\left(1+e^{x}\right)^{2}}\right) \\
+c \frac{s+(1-s) \alpha}{s} \mathcal{L}\left\{\left[\left(F_{r}-1\right)-\frac{3}{2} \frac{\varphi(x, t ; q)}{h}\right]\right. \\
\left.\frac{\partial \varphi(x, t ; q)}{\partial x}-\frac{h^{2}}{6} \frac{\partial^{3} \varphi(x, t ; q)}{\partial x^{3}}-\frac{1}{2} \frac{\partial b(x)}{\partial x}\right\} .
\end{gathered}
$$

At $\mathcal{H}(x, t)=1$, the deformation equation presented as

$$
\mathcal{L}\left[u_{m}(x, t)-k_{m} u_{m-1}(x, t)\right]=\hbar \Re_{m}\left[\vec{u}_{m-1}\right],
$$

where

$$
\begin{aligned}
& \mathfrak{R}_{m}\left[\vec{u}_{m-1}\right]=\mathcal{L}\left[u_{m-1}(x, t)\right]-\left(1-\frac{k_{m}}{n}\right) \\
& \left\{\frac{1}{s}\left(-\frac{2 e^{x}}{\left(1+e^{x}\right)^{2}}\right)\right\}+c \frac{s+(1-s) \alpha}{s} \\
& \mathcal{L}\left\{\left(F_{r}-1\right) \frac{\partial u_{m-1}}{\partial x}-\frac{3}{2 h} \sum_{i=0}^{m-1} u_{i} \frac{\partial u_{m-1-i}}{\partial x}\right. \\
& \left.-\frac{h^{2}}{6} \frac{\partial^{3} u_{m-1}}{\partial x^{3}}-\frac{1}{2} \frac{\partial b(x)}{\partial x}\right\} .
\end{aligned}
$$

Now, by the help of the initial condition, we can derive the terms of Eq. (19) as

$$
\begin{gathered}
u_{1}(x, t)=\hbar(1-\alpha+\alpha t)\left(c \left(\frac{6 \mathrm{e}^{2 x}\left(-1+\mathrm{e}^{x}\right)}{\left(1+\mathrm{e}^{x}\right)^{5} h}\right.\right. \\
-\frac{\mathrm{e}^{x}\left(-1+11 \mathrm{e}^{x}-11 \mathrm{e}^{2 x}+\mathrm{e}^{3 x}\right) h^{2}}{3\left(1+\mathrm{e}^{x}\right)^{5}}-0.025 \mathrm{e}^{-\frac{x^{2}}{4}} x \\
\left.\left.+\frac{2 \mathrm{e}^{x}\left(-1+\mathrm{e}^{x}\right)\left(-1+F_{r}\right)}{\left(1+\mathrm{e}^{x}\right)^{3}}\right)\right),
\end{gathered}
$$

Here, the existence and uniqueness are illustrated with CF operator for the considered Eq. (26) as

$$
{ }_{0}^{C F} D_{t}^{\alpha}[u(x, t)]=\mathcal{Q}(x, t, u)
$$


Now, using Eq. (31) and results derived in [46], we obtained

$$
\begin{aligned}
& u(x, t)-u(x, 0) \\
& ={ }_{0}^{C F} I_{t}^{\alpha}\left\{-c\left(\left[\left(F_{r}-1\right)-\frac{3}{2} \frac{u(x, t)}{h}\right] \frac{\partial u}{\partial x}\right.\right. \\
& \left.\left.-\frac{h^{2}}{6} \frac{\partial^{3} u}{\partial x^{3}}-\frac{1}{2} \frac{\partial b(x)}{\partial x}\right)\right\},
\end{aligned}
$$

Then we have from [41] as follows

$$
\begin{aligned}
& u(x, t)-u(x, 0)=\frac{2(1-\alpha)}{\mathcal{M}(\alpha)} \mathcal{Q}(x, t, u) \\
& +\frac{2 \alpha}{(2-\alpha) \mathcal{M}(\alpha)} \int_{0}^{t} \mathcal{Q}(x, \zeta, u) d \zeta .
\end{aligned}
$$

Theorem 3. The kernel $\mathcal{Q}$ admits the Lipschitz condition and contraction if $0 \leq$ $\left(c\left(F_{r}-1\right) \Lambda-\frac{3}{4 h} \Lambda\left(a_{1}+a_{2}\right)-\frac{h^{2}}{6} \Lambda^{3}-\frac{1}{2} \Lambda \xi\right)<$ 1 satisfies.

Proof. Consider the two functions $A$ and $A_{1}$ to prove the theorem, then

$$
\begin{aligned}
& \left\|\mathcal{Q}(x, t, u)-\mathcal{Q}\left(x, t, u_{1}\right)\right\| \\
& =\| c\left(\left(F_{r}-1\right) \frac{\partial}{\partial x}\left[u(x, t)-u\left(x, t_{1}\right)\right]\right. \\
& -\frac{3}{2 h}\left[u(x, t) \frac{\partial u(x, t)}{\partial x}-u\left(x, t_{1}\right) \frac{\partial u\left(x, t_{1}\right)}{\partial x}\right] \\
& -\frac{h^{2}}{6} \frac{\partial^{3}}{\partial x^{3}}\left[u(x, t)-u\left(x, t_{1}\right)\right]-\frac{1}{2} \frac{\partial b(x)}{\partial x} \| \\
& =\| c\left(\left(F_{r}-1\right) \frac{\partial}{\partial x}\left[u(x, t)-u\left(x, t_{1}\right)\right]\right. \\
& -\frac{3}{2 h}\left[\frac{1}{2} \frac{\partial}{\partial x}\left[u^{2}(x, t)-u^{2}\left(x, t_{1}\right)\right]\right] \\
& -\frac{h^{2}}{6} \frac{\partial^{3}}{\partial x^{3}}\left[u(x, t)-u\left(x, t_{1}\right)\right]-\frac{1}{2} \frac{\partial b(x)}{\partial x} \| \\
& \leq \| c\left(F_{r}-1\right) \Lambda-\frac{3}{4 h} \Lambda\left[u(x, t)-u\left(x, t_{1}\right)\right] \\
& -\frac{h^{2}}{6} \Lambda^{3}-\frac{1}{2} \frac{\partial b(x)}{\partial x}\|\| u(x, t)-u\left(x, t_{1}\right) \| \\
& \leq c\left(\left(F_{r}-1\right) \Lambda-\frac{3}{4 h} \Lambda\left(a_{1}+a_{2}\right)\right. \\
& \left.-\frac{h^{2}}{6} \Lambda^{3}-\frac{1}{2} \Lambda \xi\right)\left\|u(x, t)-u\left(x, t_{1}\right)\right\|,
\end{aligned}
$$

where $a_{1}=\|u\|$ and $a_{2}=\left\|u_{1}\right\|$ be the bounded function and $\|b(x)\|=\xi$ is also a bounded function. Set $\Psi=$ $c\left(\left(F_{r}-1\right) \Lambda-\frac{3}{4 h} \Lambda\left(a_{1}+a_{2}\right)-\frac{h^{2}}{6} \Lambda^{3}-\frac{1}{2} \Lambda \xi\right) \quad$ in Eq. (34), then

$$
\begin{aligned}
& \left\|\mathcal{Q}(x, t, u)-\mathcal{Q}\left(x, t, u_{1}\right)\right\| \\
& \leq \Psi\left\|u(x, t)-u\left(x, t_{1}\right)\right\| .
\end{aligned}
$$

Eq. (35) provides the Lipschitz condition for $\mathcal{Q}$. Similarly, we can see that if $0 \leq$ $c\left(\left(F_{r}-1\right) \Lambda-\frac{3}{4 h} \Lambda\left(a_{1}+a_{2}\right)-\frac{h^{2}}{6} \Lambda^{3}-\frac{1}{2} \Lambda \xi\right)<$ 1 , then it implies the contraction. By the assist of the above equations, Eq. (33) simplifies to

$$
\begin{aligned}
& u(x, t)=u(x, 0)+\frac{2(1-\alpha)}{(2-\alpha) \mathcal{M}(\alpha)} \mathcal{Q}(x, t, u) \\
& +\frac{2 \alpha}{(2-\alpha) \mathcal{M}(\alpha)} \int_{0}^{t} \mathcal{Q}(x, \zeta, u) d \zeta .
\end{aligned}
$$

Then we get the recursive form as follows

$$
\begin{aligned}
& u_{n}(x, t)=\frac{2(1-\alpha)}{(2-\alpha) \mathcal{M}(\alpha)} \mathcal{Q}\left(x, t, u_{n-1}\right) \\
& +\frac{2 \alpha}{(2-\alpha) \mathcal{M}(\alpha)} \int_{0}^{t} \mathcal{Q}\left(x, \zeta, u_{n-1}\right) d \zeta .
\end{aligned}
$$

Now, between the terms the successive difference is defined as

$$
\begin{aligned}
& \phi_{n}(x, t)=u_{n}(x, t)-u_{n-1}(x, t) \\
& =\frac{2(1-\alpha)}{(2-\alpha) \mathcal{M}(\alpha)}\left(\mathcal{Q}\left(x, t, u_{n-1}\right)-\mathcal{Q}\left(x, t, u_{n-2}\right)\right) \\
& +\frac{2 \alpha}{(2-\alpha) \mathcal{M}(\alpha)} \int_{0}^{t}\left(\mathcal{Q}\left(x, t, u_{n-1}\right)\right. \\
& \left.-\mathcal{Q}\left(x, t, u_{n-2}\right)\right) d \zeta .
\end{aligned}
$$

Notice that

$$
u_{n}(x, t)=\sum_{i=1}^{n} \phi_{i}(x, t) .
$$

Then we have

$$
\begin{aligned}
& \left\|\phi_{n}(x, t)\right\|=\left\|u_{n}(x, t)-u_{n-1}(x, t)\right\| \\
& =\| \frac{2(1-\alpha)}{(2-\alpha) \mathcal{M}(\alpha)}\left(\mathcal{Q}\left(x, t, u_{n-1}\right)\right) \\
& -\mathcal{Q}\left(x, t, u_{n-2}\right)+\frac{2 \alpha}{(2-\alpha) \mathcal{M}(\alpha)} \\
& \int_{0}^{t}\left(\mathcal{Q}\left(x, t, u_{n-1}\right)-\mathcal{Q}\left(x, t, u_{n-2}\right)\right) d \zeta \| .
\end{aligned}
$$

Application of the triangular inequality, Eq. 40 reduces to 


$$
\begin{aligned}
& \left\|\phi_{n}(x, t)\right\|=\left\|u_{n}(x, t)-u_{n-1}(x, t)\right\| \\
& =\frac{2(1-\alpha)}{(2-\alpha) \mathcal{M}(\alpha)} \\
& \left\|\left(\mathcal{Q}\left(x, t, u_{n-1}\right)-\mathcal{Q}\left(x, t, u_{n-2}\right)\right)\right\| \\
& +\frac{2 \alpha}{(2-\alpha) \mathcal{M}(\alpha)} \\
& \left\|\int_{0}^{t}\left(\mathcal{Q}\left(x, t, u_{n-1}\right)-\mathcal{Q}\left(x, t, u_{n-2}\right)\right) d \zeta\right\| .
\end{aligned}
$$

The Lipschitz condition satisfied by the kernel $t_{1}$, then

$$
\begin{aligned}
& \left\|\phi_{n}(x, t)\right\|=\left\|u_{n}(x, t)-u_{n-1}(x, t)\right\| \\
& \leq \frac{2(1-\alpha)}{(2-\alpha) \mathcal{M}(\alpha)} \Psi\left\|\phi_{(n-1)}(x, t)\right\| \\
& +\frac{2 \alpha}{(2-\alpha) \mathcal{M}(\alpha)} \Psi \int_{0}^{t}\left\|\phi_{(n-1)}(x, t)\right\| d \zeta .
\end{aligned}
$$

By the aid of the above result, we state the following result:

Theorem 4. If we have specific $t_{0}$, then the solution for Eq. (26) will exist and unique. Further, we have

$$
\frac{2(1-\alpha)}{(2-\alpha) \mathcal{M}(\alpha)} \Psi+\frac{2 \alpha}{(2-\alpha) \mathcal{M}(\alpha)} \Psi t_{0}<1 .
$$

Proof. Let $u(x, t)$ is the bounded functions admitting the Lipschitz condition. Then, we get by Eqs . 41) and 42

$$
\begin{aligned}
& \left\|\phi_{i}(x, t)\right\| \leq\left\|u_{n}(x, 0)\right\| \\
& {\left[\frac{2(1-\alpha)}{(2-\alpha) \mathcal{M}(\alpha)} \Psi+\frac{2 \alpha}{(2-\alpha) \mathcal{M}(\alpha)} \Psi t\right]^{n} .}
\end{aligned}
$$

Therefore, for the obtained solution, continuity and existence are verified. Now, to prove the Eq. (43) is a solution for Eq. (26), we consider

$$
u(x, t)-u(x, 0)=u_{n}(x, t)-\mathcal{K}_{n}(t) .
$$

Let us consider

$$
\begin{aligned}
& \left\|\mathcal{K}_{n}(t)\right\|=\| \frac{2(1-\alpha)}{(2-\alpha) \mathcal{M}(\alpha)}\left(\mathcal{Q}(x, t, u)-\mathcal{Q}\left(x, t, u_{n-1}\right)\right) \\
& +\frac{2 \alpha}{(2-\alpha) \mathcal{M}(\alpha)} \int_{0}^{t}\left(\mathcal{Q}(x, \zeta, u)-\mathcal{Q}\left(x, \zeta, u_{n-1}\right)\right) d \zeta \| \\
& \leq \frac{2(1-\alpha)}{(2-\alpha) \mathcal{M}(\alpha)}\left\|\left(\mathcal{Q}(x, t, u)-\mathcal{Q}\left(x, t, u_{n-1}\right)\right)\right\| \\
& +\frac{2 \alpha}{(2-\alpha) \mathcal{M}(\alpha)} \int_{0}^{t}\left\|\left(\mathcal{Q}(x, \zeta, u)-\mathcal{Q}\left(x, \zeta, u_{n-1}\right)\right)\right\| d \zeta
\end{aligned}
$$

$$
\begin{aligned}
& \leq \frac{2(1-\alpha)}{(2-\alpha) \mathcal{M}(\alpha)} \Psi\left\|u-u_{n-1}\right\| \\
& +\frac{2 \alpha}{(2-\alpha) \mathcal{M}(\alpha)} \Psi\left\|u-u_{n-1}\right\| t .
\end{aligned}
$$

This process gives

$$
\begin{aligned}
& \left\|\mathcal{K}_{n}(t)\right\| \\
& \leq\left(\frac{2(1-\alpha)}{(2-\alpha) \mathcal{M}(\alpha)}+\frac{2 \alpha}{(2-\alpha) \mathcal{M}(\alpha)} t\right)^{n+1} \\
& \Psi^{n+1} M
\end{aligned}
$$

Similarly, at $t_{0}$ we can obtain

$$
\begin{aligned}
& \left\|\mathcal{K}_{n}(t)\right\| \leq \\
& \left(\frac{2(1-\alpha)}{(2-\alpha) \mathcal{M}(\alpha)}+\frac{2 \alpha}{(2-\alpha) \mathcal{M}(\alpha)} t_{0}\right)^{n+1} \Psi^{n+1} M
\end{aligned}
$$

As $n \rightarrow \infty$, from Eq. (46), $\left\|\mathcal{K}_{n}(t)\right\| \rightarrow 0$ provided $\frac{2(1-\alpha)}{(2-\alpha) \mathcal{M}(\alpha)}+\frac{2 \alpha}{(2-\alpha) \mathcal{M}(\alpha)} t_{0}<1$. Next, for the solution of the projected model, we prove the uniqueness. Suppose $u^{*}(x, t)$ is another solution, then

$$
\begin{aligned}
& u(x, t)-u^{*}(x, t) \\
& =\frac{2(1-\alpha)}{(2-\alpha) \mathcal{M}(\alpha)}\left(\mathcal{Q}(x, t, u)-\mathcal{Q}\left(x, t, u^{*}\right)\right) \\
& +\frac{2 \alpha}{(2-\alpha) \mathcal{M}(\alpha)} \int_{0}^{t}\left(\mathcal{Q}(x, \zeta, u)-\mathcal{Q}\left(x, \zeta, u^{*}\right)\right) d \zeta .
\end{aligned}
$$

Now, employing the norm on the above equation we get

$$
\begin{aligned}
& \left\|u(x, t)-u^{*}(x, t)\right\| \\
& =\| \frac{2(1-\alpha)}{(2-\alpha) \mathcal{M}(\alpha)}\left(\mathcal{Q}(x, t, u)-\mathcal{Q}\left(x, t, u^{*}\right)\right) \\
& +\frac{2 \alpha}{(2-\alpha) \mathcal{M}(\alpha)} \\
& \int_{0}^{t}\left(\mathcal{Q}(x, \zeta, u)-\mathcal{Q}\left(x, \zeta, u^{*}\right)\right) d \zeta \| \\
& \leq \frac{2(1-\alpha)}{(2-\alpha) \mathcal{M}(\alpha)} \Psi\left\|u(x, t)-u^{*}(x, t)\right\| \\
& +\frac{2 \alpha}{(2-\alpha) \mathcal{M}(\alpha)} \Psi t\left\|u(x, t)-u^{*}(x, t)\right\| . \quad(48)
\end{aligned}
$$

On simplification

$$
\begin{gathered}
\left\|u(x, t)-u^{*}(x, t)\right\| \\
\left(1-\frac{2(1-\alpha)}{(2-\alpha) \mathcal{M}(\alpha)} \Psi-\frac{2 \alpha}{(2-\alpha) \mathcal{M}(\alpha)} \Psi t\right) \leq 0 .
\end{gathered}
$$

From the above condition, it is clear that $u(x, t)=u^{*}(x, t)$, if 
$\left(1-\frac{2(1-\alpha)}{(2-\alpha) \mathcal{M}(\alpha)} \Psi-\frac{2 \alpha}{(2-\alpha) \mathcal{M}(\alpha)} \Psi t\right) \geq 0$

Hence, Eq. (50) proves our required result.

\subsection{Atangana-Baleanu Sense}

Consider the equation defined in Eq. (5)

$$
\begin{aligned}
& { }_{a}^{A B C} D_{t}^{\alpha} u(x, t)+c\left(\left[\left(F_{r}-1\right)-\frac{3}{2} \frac{u(x, t)}{h}\right] \frac{\partial u}{\partial x}\right. \\
& \left.-\frac{h^{2}}{6} \frac{\partial^{3} u}{\partial x^{3}}-\frac{1}{2} \frac{\partial b(x)}{\partial x}\right)=0, \quad 0<\alpha \leq 1,
\end{aligned}
$$

with initial conditions (19). Taking $L T$ on Eq. (51) and using Eq. (19), we have

$$
\begin{array}{r}
L[u(x, t)]=\frac{1}{s}\left(-\frac{2 e^{x}}{\left(1+e^{x}\right)^{2}}\right)-\frac{c}{\mathcal{B}[\alpha]} \\
\left(1-\alpha+\frac{\alpha}{s^{\alpha}}\right) L\left\{\left[\left(F_{r}-1\right)-\frac{3}{2} \frac{u}{h}\right] \frac{\partial u}{\partial x}\right. \\
\left.-\frac{h^{2}}{6} \frac{\partial^{3} u}{\partial x^{3}}-\frac{1}{2} \frac{\partial b(x)}{\partial x}\right\} .
\end{array}
$$

Now, $\mathcal{N}$ is defined as

$$
\begin{aligned}
& \mathcal{N}[\varphi(x, t ; q)] \\
& =L[\varphi(x, t ; q)]+\frac{1}{s}\left(\frac{2 e^{x}}{\left(1+e^{x}\right)^{2}}\right) \\
& +\frac{c}{\mathcal{B}[\alpha]}\left(1-\alpha+\frac{\alpha}{s^{\alpha}}\right) \\
& L\left\{\left[\left(F_{r}-1\right)-\frac{3}{2} \frac{\varphi(x, t ; q)}{h}\right] \frac{\partial \varphi(x, t ; q)}{\partial x}\right. \\
& \left.-\frac{h^{2}}{6} \frac{\partial^{3} \varphi(x, t ; q)}{\partial x^{3}}-\frac{1}{2} \frac{\partial b(x)}{\partial x}\right\} .
\end{aligned}
$$

The deformation equation at $\mathcal{H}(x, t)=1$, is given as follows

$L\left[u_{m}(x, t)-k_{m} u_{m-1}(x, t)\right]=\hbar \Re_{m}\left[\vec{u}_{m-1}\right]$,

where

$$
\begin{aligned}
& \mathfrak{R}_{m}\left[\vec{u}_{m-1}\right] \\
& =L\left[u_{m-1}(x, t)\right]+\left(1-\frac{k_{m}}{n}\right)\left\{\frac{1}{s}\left(\frac{2 e^{x}}{\left(1+e^{x}\right)^{2}}\right)\right\} \\
& +\frac{c}{\mathcal{B}[\alpha]}\left(1-\alpha+\frac{\alpha}{s^{\alpha}}\right) L\left\{\left(F_{r}-1\right) \frac{\partial u_{m-1}}{\partial x}\right. \\
& \left.-\frac{3}{2 h} \sum_{i=0}^{m-1} u_{i} \frac{\partial u_{m-1-i}}{\partial x}-\frac{h^{2}}{6} \frac{\partial^{3} u_{m-1}}{\partial x^{3}}-\frac{1}{2} \frac{\partial b(x)}{\partial x}\right\} .
\end{aligned}
$$

Now, by the help of the initial condition, we can derive

$$
u_{1}(x, t)=\hbar\left(1-\alpha+\frac{\alpha t^{\alpha}}{\Gamma[\alpha+1]}\right)
$$

$$
\begin{gathered}
\left(c \left(\frac{6 \mathrm{e}^{2 x}\left(-1+\mathrm{e}^{x}\right)}{\left(1+\mathrm{e}^{x}\right)^{5} h}-\frac{\mathrm{e}^{x}\left(-1+11 \mathrm{e}^{x}-11 \mathrm{e}^{2 x}+\mathrm{e}^{3 x}\right) h^{2}}{3\left(1+\mathrm{e}^{x}\right)^{5}}\right.\right. \\
\left.\left.-0.025 \mathrm{e}^{-\frac{x^{2}}{4}} x+\frac{2 \mathrm{e}^{x}\left(-1+\mathrm{e}^{x}\right)\left(-1+F_{r}\right)}{\left(1+\mathrm{e}^{x}\right)^{3}}\right)\right),
\end{gathered}
$$

In the segment, the existence and uniqueness are illustrated for the considered equation associated with $\mathrm{AB}$ operator. We have from Eq. (51),

$$
{ }_{a}^{A B C} D_{t}^{\alpha} u(x, t)=\mathcal{G}(x, t, u),
$$

and the above equation is considered as

$$
{ }_{0}^{A B C} D_{t}^{\alpha}[u(x, t)]=\mathcal{G}(x, t, u) .
$$

We have from Eq. (57) and Theorem 2

$$
\begin{aligned}
& u(x, t)-u(x, 0)=\frac{(1-\alpha)}{\mathcal{B}(\alpha)} \mathcal{G}(x, t, u) \\
& +\frac{\alpha}{\mathcal{B}(\alpha) \Gamma(\alpha)} \int_{0}^{t} \mathcal{G}(x, \zeta, u)(t-\zeta)^{\alpha-1} d \zeta .
\end{aligned}
$$

Theorem 5. The kernel $\mathcal{G}$ admits the Lipschitz condition and contraction if $0 \leq$ $\left(c\left(\left(F_{r}-1\right) \delta-\frac{3}{4 h} \delta(a+b)-\frac{h^{2}}{6} \delta^{3}-\frac{1}{2} \delta \xi\right)\right)<1$ satisfies.

Proof. To prove the theorem, let us consider the two functions $u$ and $u_{1}$, then

$$
\begin{gathered}
\left\|\mathcal{G}(x, t, u)-\mathcal{G}\left(x, t, u_{1}\right)\right\| \\
=\| c\left(\left(F_{r}-1\right) \frac{\partial}{\partial x}\left[u(x, t)-u\left(x, t_{1}\right)\right]\right. \\
-\frac{3}{2 h}\left[u(x, t) \frac{\partial u(x, t)}{\partial x}-u\left(x, t_{1}\right) \frac{\partial u\left(x, t_{1}\right)}{\partial x}\right] \\
-\frac{h^{2}}{6} \frac{\partial^{3}}{\partial x^{3}}\left[u(x, t)-u\left(x, t_{1}\right)\right]-\frac{1}{2} \frac{\partial b(x)}{\partial x} \| \\
\leq \| c\left(F_{r}-1\right) \delta-\frac{3}{4 h} \delta\left[u(x, t)-u\left(x, t_{1}\right)\right] \\
-\frac{h^{2}}{6} \delta^{3}-\frac{1}{2} \frac{\partial b(x)}{\partial x}\|\| u(x, t)-u\left(x, t_{1}\right) \| \\
\leq c\left(\left(F_{r}-1\right) \delta-\frac{3}{4 h} \delta(a+b)-\frac{h^{2}}{6} \delta^{3}-\frac{1}{2} \delta \xi\right) \\
\times\left\|u(x, t)-u\left(x, t_{1}\right)\right\|,
\end{gathered}
$$

where $a=\|u\|, \quad b=\left\|u_{1}\right\|$ (since $u$ and $u_{1}$ are the bounded functions) and $\|b(x)\|=\xi$ is also a bounded function. Putting $\eta=$ 
$c\left(\left(F_{r}-1\right) \delta-\frac{3}{4 h} \delta(a+b)-\frac{h^{2}}{6} \delta^{3}-\frac{1}{2} \delta \xi\right)$ in Eq. 59 , then

$$
\begin{aligned}
& \left\|\mathcal{G}(x, t, u)-\mathcal{G}\left(x, t, u_{1}\right)\right\| \\
& \leq \eta\left\|u(x, t)-u\left(x, t_{1}\right)\right\| .
\end{aligned}
$$

By the assist of forgoing relation, the Lipschitz condition is achieved for $\mathcal{G}$. Further, we can see that if $0 \leq\left(\frac{\sigma^{2}}{2} \delta^{2}+\lambda\left(a^{2}+b^{2}+a b\right)\right)<1$, which leads to contraction. The recursive form of Eq. (60) is presented as

$$
\begin{aligned}
& u_{n}(x, t)=\frac{(1-\alpha)}{\mathcal{B}(\alpha)} \mathcal{G}\left(x, t, u_{n-1}\right) \\
& +\frac{\alpha}{\mathcal{B}(\alpha) \Gamma(\alpha)} \int_{0}^{t} \mathcal{G}\left(x, \zeta, u_{n-1}\right)(t-\zeta)^{\alpha-1} d \zeta,
\end{aligned}
$$

and initial condition

$$
u(x, 0)=u_{0}(x, t) .
$$

The successive difference between the terms is presented as

$$
\begin{array}{r}
\phi_{n}(x, t)=u_{n}(x, t)-u_{n-1}(x, t) \\
=\frac{(1-\alpha)}{\mathcal{B}(\alpha)}\left(\mathcal{G}_{1}\left(x, t, u_{n-1}\right)-\mathcal{G}\left(x, t, u_{n-2}\right)\right) \\
+\frac{\alpha}{\mathcal{B}(\alpha) \Gamma(\alpha)} \int_{0}^{t} \mathcal{G}\left(x, \zeta, u_{n-1}\right)(t-\zeta)^{\alpha-1} d \zeta .
\end{array}
$$

Notice that

$$
u_{n}(x, t)=\sum_{i=1}^{n} \phi_{i}(x, t) .
$$

Plugging the norm on the Eq. (63), and by the assist of Eq. (58), we get

$$
\begin{aligned}
& \left\|\phi_{n}(x, t)\right\| \leq \frac{(1-\alpha)}{\mathcal{B}(\alpha)} \eta\left\|\phi_{(n-1)}(x, t)\right\| \\
& +\frac{\alpha}{\mathcal{B}(\alpha) \Gamma(\alpha)} \eta \int_{0}^{t}\left\|\phi_{(n-1)}(x, \zeta)\right\| d \zeta .
\end{aligned}
$$

By the assist of the above result, we prove the following theorem.

Theorem 6. The solution for Eq. (51) will exist and unique if there exist a $t_{0}$ then

$$
\frac{(1-\alpha)}{\mathcal{B}(\alpha)} \eta+\frac{\alpha}{\mathcal{B}(\alpha) \Gamma(\alpha)} \eta<1 .
$$

Proof. Let us consider the bounded function $u(x, t)$ satisfying the Lipschitz condition. Then, we get by Eq. (63) and Eq. 65), one can get

$$
\begin{aligned}
\|\phi(x, t)\| \leq & \left\|u_{n}(x, 0)\right\| \\
& {\left[\frac{(1-\alpha)}{\mathcal{B}(\alpha)} \eta+\frac{\alpha}{\mathcal{B}(\alpha) \Gamma(\alpha)} \eta\right]^{n} . }
\end{aligned}
$$

Therefore, for the obtained solutions, continuity and existence are verified. Now, to prove the Eq. (66) is a solution for Eq. (51), we consider

$$
u(x, t)-u(x, 0)=u_{n}(x, t)-\mathcal{K}_{n}(x, t) .
$$

Now, we consider

$$
\begin{gathered}
\left\|\mathcal{K}_{n}(x, t)\right\|=\| \frac{(1-\alpha)}{\mathcal{B}(\alpha)}\left(\mathcal{G}(x, t, u)-\mathcal{G}\left(x, t, u_{n-1}\right)\right) \\
\quad+\frac{\alpha}{\mathcal{B}(\alpha) \Gamma(\alpha)} \\
\int_{0}^{t}(t-\zeta)^{\mu-1}\left(\mathcal{G}(x, \zeta, u)-\mathcal{G}\left(x, \zeta, u_{n-1}\right)\right) d \zeta \| \\
\leq \frac{(1-\alpha)}{\mathcal{B}(\alpha)}\left\|\mathcal{G}(x, \zeta, u)-\mathcal{G}\left(x, \zeta, u_{n-1}\right)\right\| \\
+\frac{\alpha}{\mathcal{B}(\alpha) \Gamma(\alpha)} \int_{0}^{t}\left\|\mathcal{G}(x, \zeta, u)-\mathcal{G}\left(x, \zeta, u_{n-1}\right)\right\| d \zeta \\
\leq \frac{(1-\alpha)}{\mathcal{B}(\alpha)} \eta_{1}\left\|u-u_{n-1}\right\| \\
+\frac{\alpha}{\mathcal{B}(\alpha) \Gamma(\alpha)} \eta_{1}\left\|u-u_{n-1}\right\| t .
\end{gathered}
$$

Similarly, at $t_{0}$ we can obtain

$$
\begin{aligned}
\left\|\mathcal{K}_{n}(x, t)\right\| \leq & \left(\frac{(1-\alpha)}{\mathcal{B}(\alpha)}+\frac{\alpha t_{0}}{\mathcal{B}(\alpha) \Gamma(\alpha)}\right)^{n+1} \\
& \eta^{n+1} M .
\end{aligned}
$$

As $n$ tends to $\infty$, then $\left\|\mathcal{K}_{n}(x, t)\right\|$ approaches to 0 with respect to Eq. 69.

$$
\begin{aligned}
& u(x, t)-u^{*}(x, t) \\
& =\frac{(1-\alpha)}{\mathcal{B}(\alpha)}\left(\mathcal{G}(x, t, u)-\mathcal{G}\left(x, t, u^{*}\right)\right) \\
& +\frac{\alpha}{\mathcal{B}(\alpha) \Gamma(\alpha)} \int_{0}^{t}\left(\mathcal{G}(x, \zeta, u)-\mathcal{G}\left(x, \zeta, u^{*}\right)\right) d \zeta .
\end{aligned}
$$

The Eq. (70) simplifies on applying norm,

$$
\begin{aligned}
& \left\|u(x, t)-u^{*}(x, t)\right\| \\
& =\| \frac{(1-\alpha)}{\mathcal{B}(\alpha)}\left(\mathcal{G}(x, t, u)-\mathcal{G}\left(x, t, u^{*}\right)\right) \\
& +\frac{\alpha}{\mathcal{B}(\alpha) \Gamma(\alpha)} \int_{0}^{t}\left(\mathcal{G}(x, \zeta, u)-\mathcal{G}\left(x, \zeta, u^{*}\right)\right) d \zeta \| \\
& \leq \frac{(1-\alpha)}{\mathcal{B}(\alpha)} \eta\left\|u(x, t)-u^{*}(x, t)\right\| \\
& +\frac{\alpha}{\mathcal{B}(\alpha) \Gamma(\alpha)} \eta t\left\|u(x, t)-u^{*}(x, t)\right\| .
\end{aligned}
$$

On simplification

$$
\begin{aligned}
& \left\|u(x, t)-u^{*}(x, t)\right\| \\
& \left(1-\frac{\alpha}{\mathcal{B}(\alpha) \Gamma(\alpha)} \eta t-\frac{(1-\alpha)}{\mathcal{B}(\alpha)} \eta\right) \leq 0 .
\end{aligned}
$$

From the above condition, it is clear that $u(x, t)=u^{*}(x, t)$, if

$$
\left(1-\frac{\alpha}{\mathcal{B}(\alpha) \Gamma(\alpha)} \eta t-\frac{(1-\alpha)}{\mathcal{B}(\alpha)} \eta\right) \geq 0 .
$$




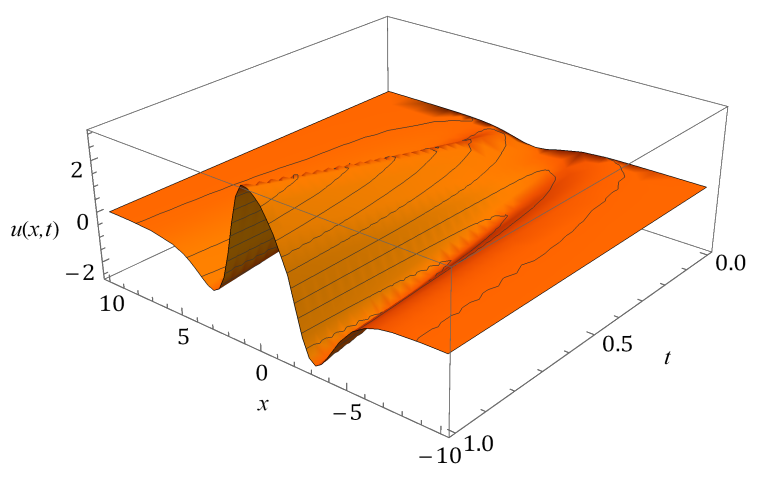

(a)

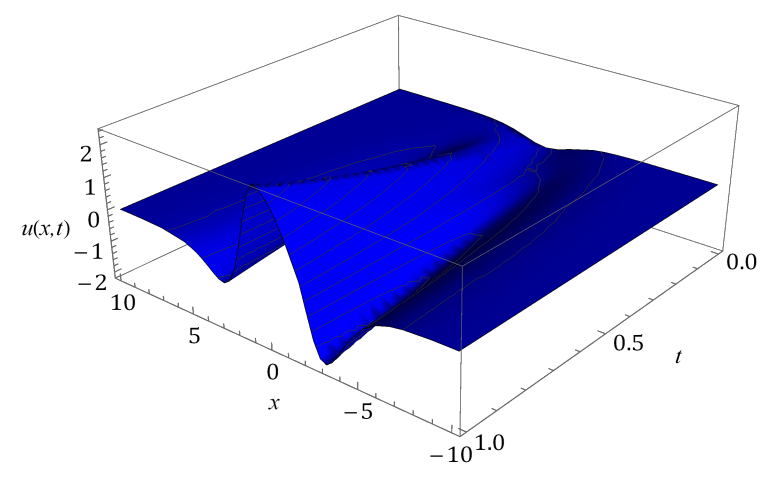

(b)

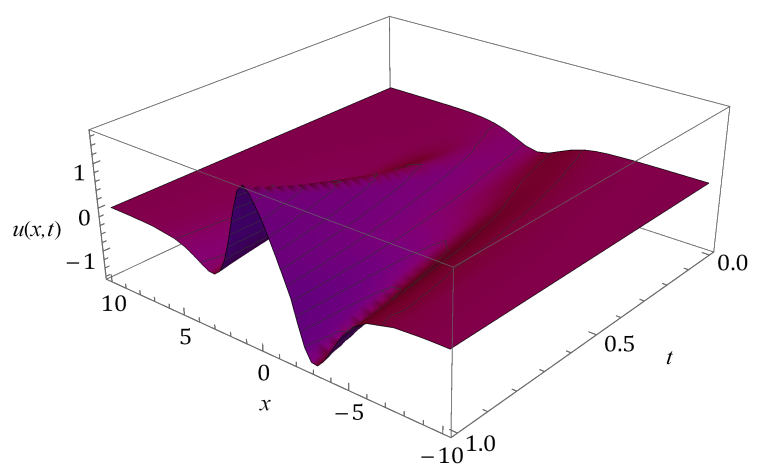

(c)

Figure 2. Surfaces of $q$-HATT solution for $(\boldsymbol{a})$ Caputo, $(\boldsymbol{b}) \mathrm{CF}$ and $(\boldsymbol{c})$ AB fractional operator at $n=1, \hbar=-1, \beta=2, \alpha=1$ and $F_{r}=-1$.
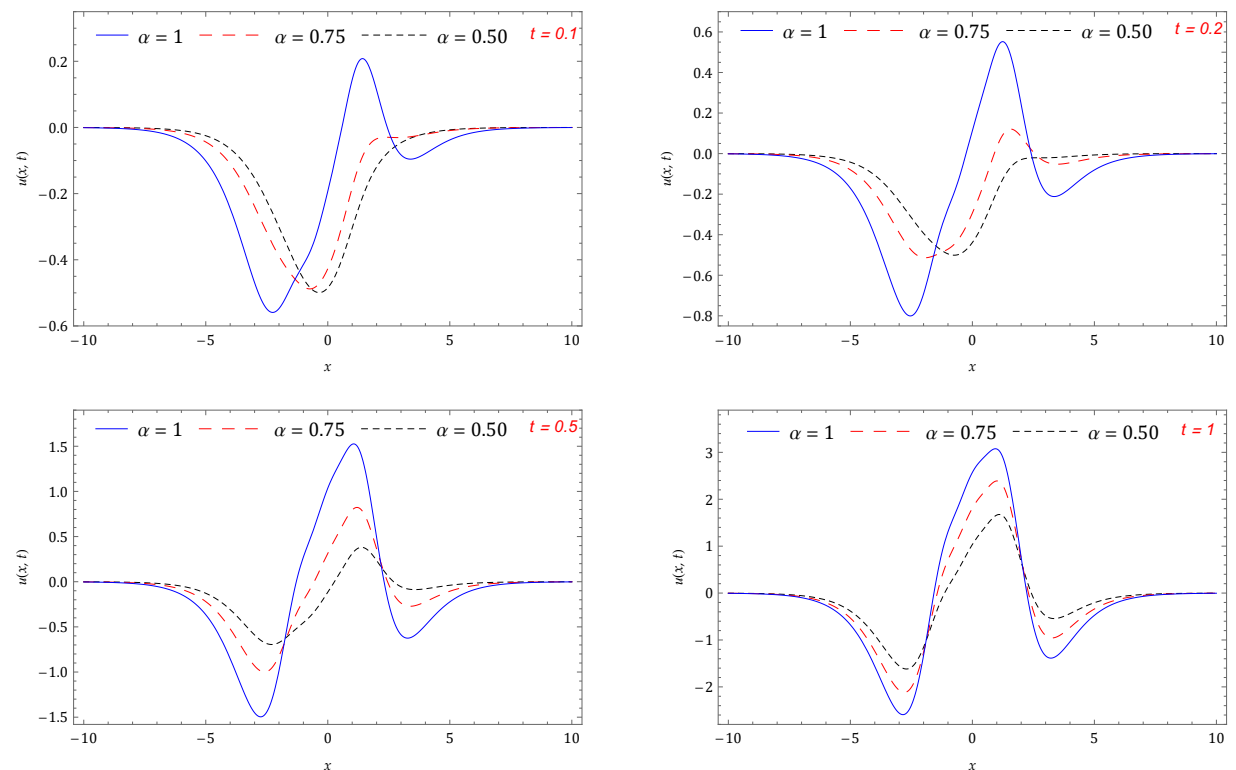

Figure 3. Response of obtained solution with distinct $\alpha$ and time at $n=1, \hbar=-1, \beta=2$ and $F_{r}=-1$.

Hence, Eq. $\quad(73)$ evidence required consequence.

\section{Results and discussion}

In this section, we consider two cases as mentioned above to analyze the hired model with a hole, and presented in Figure 1. In the first case 

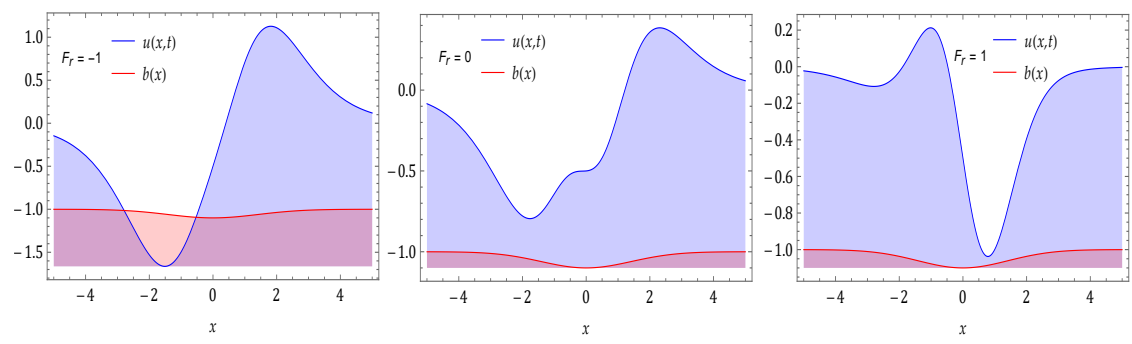

Figure 4. Nature of water elevation with $b(x)$ at $n=1, \hbar=-1, \beta=2, t=1.5$ and $\alpha=1$.

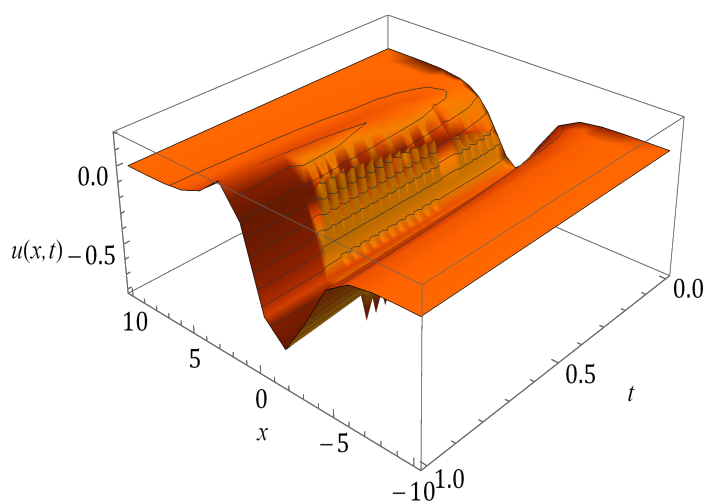

(a)

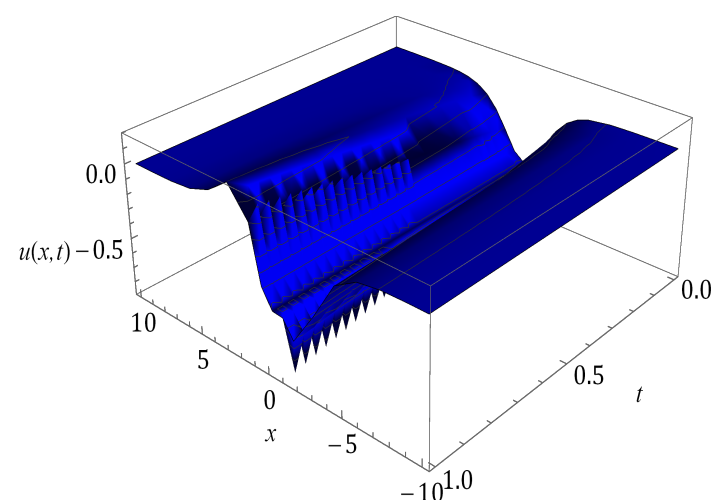

(b)

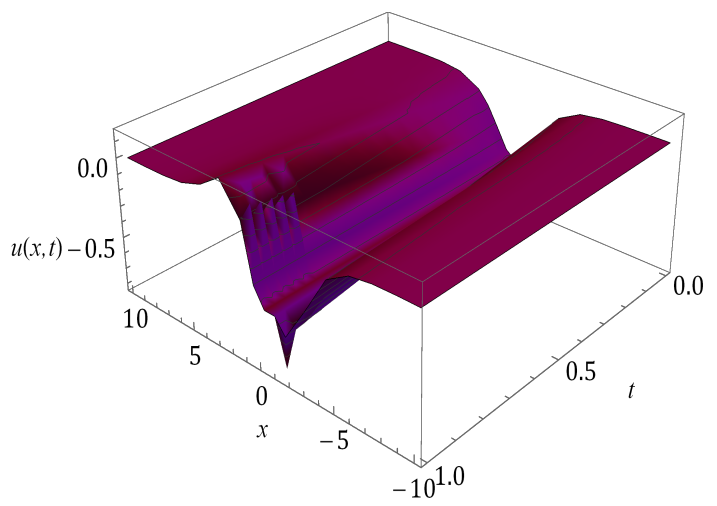

(c)

Figure 5. Surfaces of $q$-HATT solution for (a) Caputo, (b) CF and (c) AB fractional operator at $n=1, \hbar=-1, \beta=8, \alpha=1$ and $F_{r}=-1$.

for $\beta=2$, the behaviour of $b(x)$ is a lock-like reciprocal of bell-shape and also sharp at the bottom. For the second case (i.e., $\beta=8$ ), the hole at the bottom is more flattened and wider. In the present investigation, we consider constant wave speed $c \approx \sqrt{g \times h}=\sqrt{9.8}$ with a mean water depth of the sea $h=1$. For $\beta=2$, the nature of archived results for the FF-KdV equation with different distinct fractional operator and fractional-order is captured respectively in Figures 2 and 3. In Figure 3 we can observe that at $x=-2$ and 2 the behaviour of water evaluation is overlapped for different value of $\alpha$ and moreover the change in time shows stimulating variation in the behaviours. The nature of the water elevation with sea bed topography with $\beta=2$ and 8 are presented in Figures 4 and 7 for different Froude number in order to understand the importance of $b(x)$ and $\beta$ in the obtained solution at the particular values of the time. In the same manner, for $n=8$ surfaces for an obtained solution with a distinct fractional operator is cited in Figure 5. The response of $q$-HATT solution for FF-KdV equation with distinct $\alpha$ is dissipated in Figure 6 for $\beta=8$. In this case, also we can notice the huge change in the behaviours with a small change in time with fractional order.

The considered method is highly noticeable for the parameters associated with the algorithm and which help to make more convergence (they are proposed based on the topological concept). To illustrate the nature of the solution obtained with homotopy parameter $(\hbar)$, the $\hbar$-curves are plotted 

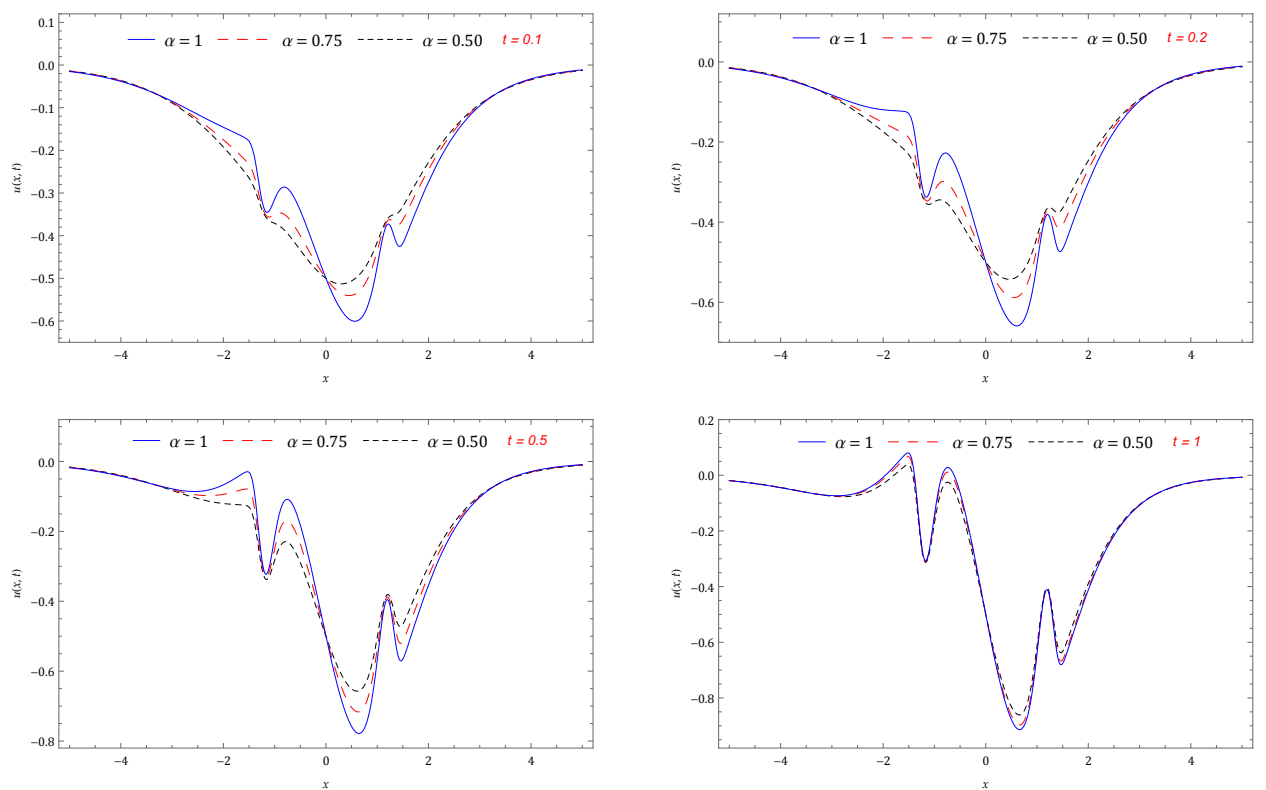

Figure 6. Response of obtained solution with distinct $\alpha$ and time at $n=1, \hbar=-1, \beta=8$ and $F_{r}=-1$.
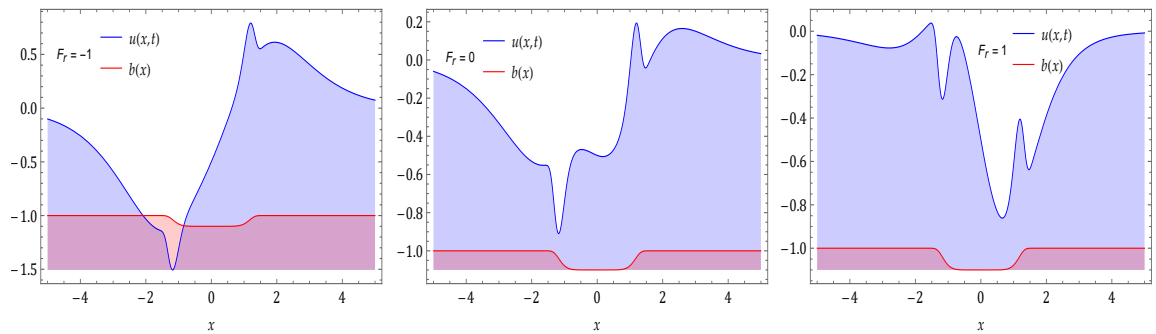

Figure 7. Nature of water elevation with $b(x)$ at $n=1, \hbar=-1, \beta=8$ and $\alpha=1$.

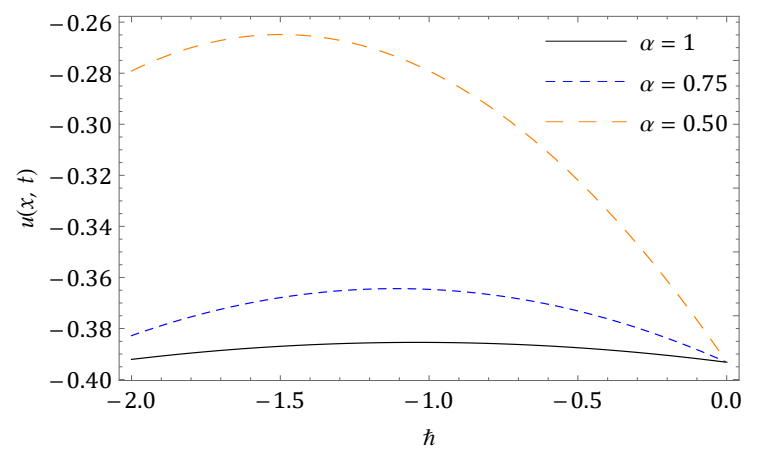

(a)

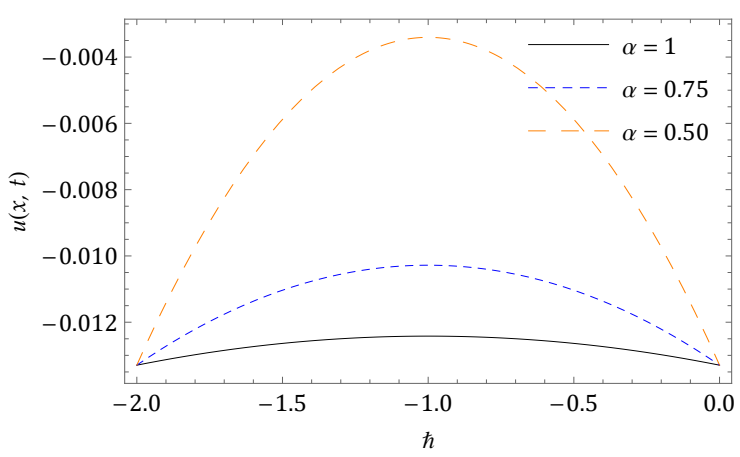

(b)

Figure 8. $\hbar$-curves for $q$-HATT solution with distinct $\alpha$ when $n=1$ and $t=0.01$ with (a) $\beta=2$ at $x=1$ and $(b) \beta=8$ at $x=5$.

with different $\alpha$ for both cases (i.e., $\beta=2$ and 8 ) and are respectively captured in Figure 8. Line flat segment designates the convergence providence of the solution.

\section{Conclusion}

In this study, the $q$-HATT is applied lucratively to the analyzed effect of parameters associated with the method (rigid bottom topography and Froude number) by finding the solution for an arbitrary order shallow water forced $\mathrm{KdV}$ equation describing the free surface critical flow over a hole. The derived results show the effect of rigid bottom topography and Froude number with change in time and space with different fractional order. By using the considered model, two distinct kinds of hole are analyzed and which shows that for $\beta=2$ 
exhibits a hole in inverse-bell shape and at $\beta=8$ shows a hole has a sharp edge on two-sides and also it has a flattened base. The condition is derived for the considered model to illustrate the existence and uniqueness within the frame of fixedpoint theory using Banach space. The effect of three fractional operators is presented and other effects are illustrated with respect to the Caputo operator. These fractional operators are playing a vital role in generalizing the models associated with power-law distribution, kernel singular, and non-local and non-singular (respectively, Caputo, $\mathrm{CF}$ and $\mathrm{AB}$ operators). Finally, the present study is to demonstrate the effect of fractional order, parameters associated with models as well as methods with their corresponding consequences.

\section{References}

[1] Liouville, J. (1832). Memoire surquelques questions de geometrieet de mecanique, et sur un nouveau genre de calcul pour resoudreces questions, J. Ecole. Polytech., 13, 1-69.

[2] Riemann, G.F.B. (1896). Versuch Einer Allgemeinen Auffassung der Integration und Differentiation, Gesammelte Mathematische Werke, Leipzig.

[3] Caputo, M. (1969). Elasticita e Dissipazione, Zanichelli, Bologna.

[4] Miller, K.S. \& Ross, B. (1993). An introduction to fractional calculus and fractional differential equations, A Wiley, New York.

[5] Podlubny, I. (1999) Fractional Differential Equations, Academic Press, New York.

[6] Kilbas, A.A., Srivastava, H.M. \& Trujillo, J.J. (2006). Theory and applications of fractional differential equations, Elsevier, Amsterdam.

[7] Baleanu, D. Guvenc, Z.B. \& Tenreiro Machado, J.A. (2010). New trends in nanotechnology and fractional calculus applications, Springer Dordrecht Heidelberg, London New York.

[8] Naik, P. A., Yavuz, M., Qureshi, S., Zu, J., \& Townley, S. (2020). Modeling and analysis of COVID-19 epidemics with treatment in fractional derivatives using real data from Pakistan. The European Physical Journal Plus, 135(10), 1-42.

[9] Evirgen, F., \& Yavuz, M. (2018). An alternative approach for nonlinear optimization problem with Caputo-Fabrizio derivative. In ITM Web of Conferences (Vol. 22, p. 01009). EDP Sciences.

[10] Yokus, A. (2021). Construction of different types of traveling wave solutions of the relativistic wave equation associated with the Schrödinger equation. Mathematical Modelling and Numerical Simulation with Applications, 1(1), 24-31.

[11] Yavuz, M., Coşar, F. Ö., Günay, F., \& Özdemir, F. N. (2021). A new mathematical modeling of the COVID-19 pandemic including the vaccination campaign. Open Journal of Modelling and Simulation, 9(3), 299-321.

[12] Uçar, E., Uçar, S., Evirgen, F., \& Özdemir, N. (2021). A Fractional SAIDR Model in the Frame of Atangana-Baleanu Derivative. Fractal and Fractional, 5(2), 32.

[13] Kumar, P., Erturk, V. S., Banerjee, R., Yavuz, M., \& Govindaraj, V. (2021). Fractional modeling of plankton-oxygen dynamics under climate change by the application of a recent numerical algorithm. Physica Scripta, 96(12), 124044.

[14] Daşbaşı, B. (2021). Stability analysis of an incommensurate fractional-order SIR model. Mathematical Modelling and Numerical Simulation with Applications, 1(1), 44-55.

[15] Uçar, E., Uçar, S., Evirgen, F., \& Özdemir, N. (2021). Investigation of E-Cigarette Smoking Model with Mittag-Leffler Kernel. Foundations of Computing and Decision Sciences, 46(1), 97-109.

[16] Akinyemi, L. et al., (2021), Novel soliton solutions of four sets of generalized (2+1)-dimensional BoussinesqKadomtsev-Petviashvili-like equations, Modern Physics Letters B, 2150530, DOI: $10.1142 / \mathrm{s} 0217984921505308$.

[17] Veeresha, P. (2021). A numerical approach to the coupled atmospheric ocean model using a fractional operator. Mathematical Modelling and Numerical Simulation with Applications, 1(1), 1-10.

[18] Baishya, C. \& Veeresha, P. (2021), Laguerre polynomial-based operational matrix of integration for solving fractional differential equations with non-singular kernel, Proceeding of the Royal Society A, 477(2253).

[19] Baishya, C., Achar, S.J., Veeresha, P. \& Prakasha, D.G. (2021), Dynamics of a fractional epidemiological model with disease infection in both the populations, Chaos, 31, 043130.

[20] Baskonus, H.M., T. A. Sulaiman, H. Bulut, On the new wave behavior to the Klein-Gordon-Zakharov equations in plasma physics, Indian J. Phys. 93 (3) (2019) 393399.

[21] Yao, S.W., Ilhan, E., Veeresha, P. \& Baskonus, H.M. (2021). A powerful iterative approach for quintic complex 
Ginzburg-Landau equation within the frame of fractional operator, Fractals, DOI: 10.1142/S0218348X21400235.

[22] Veeresha, P., Prakasha, D.G. \& Baleanu, D. (2019). An efficient numerical technique for the nonlinear fractional KolmogorovPetrovskii-Piskunov equation, Mathematics, 7(3), 1-18. DOI:10.3390/math7030265.

[23] Ali, M.R., Ma, W.X. \& Sadat, R. (2021). Lie symmetry analysis and invariant solutions for $(2+1)$ dimensional Bogoyavlensky-Konopelchenko equation with variable-coefficient in wave propagation. Journal of Ocean Engineering and Science, DOI: $10.1016 /$ j.joes.2021.08.006.

[24] Akinyemi, L., \& Iyiola, O. S. (2021). Analytical Study of $(3+1)$-Dimensional FractionalReaction Diffusion Trimolecular Models. International Journal of Applied and Computational Mathematics, 7(3), 1-24.

[25] Veeresha, P. \& Baleanu, D. (2021). A unifying computational framework for fractional Gross-Pitaevskii equations. Physica Scripta, 96(125010).

[26] Ali, M.R., Sadat, R. \& Ma, W.X. (2021). Investigation of new solutions for an extended (2+1)-dimensional Calogero-BogoyavlenskiiSchif equation. Frontiers of Mathematics in China, 16(4), 925-936.

[27] Akinyemi, L., \& Iyiola, O.S. (2020). A reliable technique to study nonlinear timefractional coupled Korteweg-de Vries equations. Advances in Difference equations, 2020(1), 1-27.

[28] Ali, M.R. \& Ma, W.X. (2020). New exact solutions of Bratu Gelfand model in two dimensions using Lie symmetry analysis. Chinese Journal of Physics, 65, 198-206.

[29] Akinyemi, L., et al. (2021). Novel approach to the analysis of fifth-order weakly nonlocal fractional Schrodinger equation with Caputo derivative. Results in Physics, 104958, DOI: 10.1016/j.rinp.2021.104958.

[30] Safare, K.M. et al. (2021). A mathematical analysis of ongoing outbreak COVID-19 in India through nonsingular derivative. Numerical Methods for Partial Differential Equations, 37(2), 1282-1298.

[31] Akinyemi, L., Veeresha, P., \& Ajibola, S.O. (2021). Numerical simulation for coupled nonlinear Schrödinger-Korteweg-de Vries and Maccari systems of equations. Modern Physics Letters B, 2150339.
[32] Hammouch, Z., Yavuz, M., \& Özdemir, N. (2021). Numerical solutions and synchronization of a variable-order fractional chaotic system. Mathematical Modelling and Numerical Simulation with Applications (MMNSA), 1(1), 11-23.

[33] Veeresha, P., Ilhan, E., Prakasha, D.G., Baskonus, H.M. \& Gao, W. (2021). Regarding on the fractional mathematical model of Tumour invasion and metastasis. Computer Modeling in Engineering 83 Sciences, 127(3), 1013-1036.

[34] Akinyemi, L., \& Huseen, S.N. (2020). A powerful approach to study the new modified coupled Korteweg-de Vries system. Mathematics and Computers in Simulation, 177, 556-567.

[35] Ali, M.R., \& Sadat, R. (2021). Construction of Lump and optical solitons solutions for $(3+$ 1) model for the propagation of nonlinear dispersive waves in inhomogeneous media. Optical and Quantum Electronics, 53(5), 1-13.

[36] Baishya, C. (2021). An operational matrix based on the Independence polynomial of a complete bipartite graph for the Caputo fractional derivative. SeMA Journal, 1-19.

[37] Şenol, M., Iyiola, O.S., Daei Kasmaei, H., \& Akinyemi, L. (2019). Efficient analytical techniques for solving time-fractional nonlinear coupled Jaulent-Miodek system with energydependent Schrödinger potential. Advances in Difference Equations, 2019(1), 1-21.

[38] Veeresha, P., Ilhan, E., Prakasha, D.G., Baskonus, H.M. \& Gao, W. (2021). A new numerical investigation of fractional order susceptible-infected-recovered epidemic model of childhood disease. Alexandria Engineering Journal, DOI: 10.1016/j.aej.2021.07.015.

[39] Ali, M.R., \& Sadat, R. (2021). Lie symmetry analysis, new group invariant for the $(3+1)$ dimensional and variable coefficients for liquids with gas bubbles models. Chinese Journal of Physics, 71, 539-547.

[40] Akinyemi, L., Şenol, M., \& Huseen, S. N. (2021). Modified homotopy methods for generalized fractional perturbed Zakharov-Kuznetsov equation in dusty plasma. Advances in Difference Equations, 2021(1), 127.

[41] Veeresha, P. \& Prakasha, D.G. (2021). Novel approach for modified forms of Camassa-Holm and Degasperis-Procesi equations using fractional operator. Communications in Theoretical Physics, 72(10). 
[42] Dias, F. \& Vanden-Broeck, J.M. (2002). Generalized critical free-surface flows, J. Eng. Math. 42, 291-301.

[43] Shen, S.S. (1995). On the accuracy of the stationary forced Korteweg-De Vries equation as a model equation for flows over a bump, $Q$. Appl. Math. 53, 701-719.

[44] Camassa, R. \& Wu, T. (1991), Stability of forced solitary waves, Philos. Trans. R. Soc. Lond. A, 337, 429-466.

[45] Zabuski, N.J. \& Kruskal, M.D. (1965). Interaction of solitons in a collisionless plasma and the recurrence of initial states, Phys. Rev. Lett., 15, 240-243.

[46] Crighton, D.G. (1995). Applications of KdV, Acta Appl. Math., 39, 39-67.

[47] Hereman, W. (2012), Shallow Water Waves and Solitary Waves, In Mathematics of Complexity and Dynamical Systems; Meyers, $R$., Ed.; Springer: New York, USA.

[48] Yao-Tsu Wu, T. (1987). Generation of upstream advancing solitons by moving disturbances, J. Fluid Mech., 184, 75-99.

[49] David, V.D., Aziz, Z.A. \& Salah, F. (2016). Analytical approximate solution for the forced Korteweg-de Vries (FKdV) on critical flow over a hole using homotopy analysis method, Jurnal Teknologi (Sciences $\&$ Engineering), 78(3-2), 107-112.

[50] Caputo, M. \& Fabrizio, M. (2016). A new definition of fractional derivative without singular kernel, Progress in Fractional Differentiation and Applications, 1(2), 73-85.

[51] Atangana, A. \& Baleanu, D. (2016). New fractional derivatives with non-local and nonsingular kernel theory and application to heat transfer model, Thermal Science, 20, 763-769.

[52] Jun-Xiao, Z. \& Bo-Ling, G. (2009), Analytic solutions to forced KdV equation. Commun. Theor. Phys., 52, 279-283.

[53] Milewski, P.A. (2014). The forced KortewegDe Vries equation as a model for waves generated by topography. Cubo, 6(4), 33-51.

[54] David, V.D., Salah, F., Nazari, M. \& Aziz, Z.A. (2013). Approximate analytical solution for the forced Korteweg-de Vries equation. Journal of Applied Mathematics, 1-9, DOI: $10.1155 / 2013 / 795818$.

[55] Lee, S. (2018). Dynamics of trapped solitary waves for the forced KdV equation, Symmetry 10(129), 1-13, DOI: 10.3390/sym10050129.

[56] Tay, K.G., Tiong, W.K., Choy, Y.Y. \& Ong, C.T. (2017). Method of lines and pseudospectral solutions of the forced Korteweg-De Vries equation with variable coefficients arises in elastic tube. International Journal of Pure and Applied Mathematics, 116(4), 985-999.

[57] Liao, S.J. (1997). Homotopy analysis method and its applications in mathematics, J. Basic Sci. Eng., 5(2), 111-125.

[58] Liao, S.J. (1998). Homotopy analysis method: a new analytic method for nonlinear problems, Appl. Math. Mech., 19, 957-962.

[59] Singh, J., Kumar, D. \& Swroop, R. (2016). Numerical solution of time- and spacefractional coupled Burgers' equations via homotopy algorithm. Alexandria Eng. J., 55(2), 1753-1763.

[60] Srivastava, H.M., Kumar, D. \& Singh, J., An efficient analytical technique for fractional model of vibration equation. Appl. Math. Model., 45, 192-204.

[61] Veeresha, P., Prakasha, D.G., Singh, J., Kumar, D. \& Baleanu, D. (2020). Fractional Klein-Gordon-Schrödinger equations with Mittag-Leffler memory. Chinese J. Phy., 68, 65-78.

[62] Prakasha, D.G., et al. (2021). An efficient computational technique for timefractional Kaup-Kupershmidt equation. $\mathrm{Nu}$ merical Methods for Partial Differential Equations, 37(2), 1299-1316.

[63] Veeresha, P. \& Prakasha, D.G. (2019). A novel technique for $(2+1)$-dimensional timefractional coupled Burgers equations. Mathematics and Computers in Simulation, 166, 324-345.

[64] D. Kumar, R.P. Agarwal, J. Singh, A modified numerical scheme and convergence analysis for fractional model of Lienard's equation, J. Comput. Appl. Math. 399 (2018) 405-413.

[65] Veeresha, P., Ilhan, E. \& Baskonus, H.M. (2021). Fractional approach for analysis of the model describing wind-influenced projectile motion. Physica Scripta, 96(7), 075209.

[66] Veeresha, P. \& Prakasha, D.G. (2021). Solution for fractional Kuramoto-Sivashinsky equation using novel computational technique. International Journal of Applied and Computational Mathematics, 7(33).

[67] Okposo, N.I., Veeresha, P. \& Okposo, E.N. (2021). Solutions for time-fractional coupled nonlinear Schrödinger equations arising in optical solitons. Chinese Journal of Physics, DOI: 10.1016/j.cjph.2021.10.014.

[68] Losada, J. \& Nieto, J.J. (2015), Properties of the new fractional derivative without singular Kernel, Progr. Fract. Differ. Appl., 1, 87-92.

[69] Atangana, A. \& Koca, I. Chaos in a simple nonlinear system with Atangana-Baleanu 
derivatives with fractional order. Chaos Solitons Fractals, 89, 447-454.

Pundikala Veeresha is an Assistant Professor in the Department of Mathematics, CHRIST (Deemed to be University), Bengaluru. He completed his Master's Degree from Davangere University, Davangere, and his Ph.D. from Karnatak University, Dharwad. His areas of research interest includes Fractional Calculus, Mathematical Modelling, Numerical and Analytical Methods, and Mathematical Physics. He has been the author of more than 70 research articles published in highly reputed journals.

(1) https://orcid.org/0000-0002-4468-3048

Mehmet Yavuz received his Ph.D. in Mathematics from Balıkesir University, Turkey. He visited the University of Exeter, U.K. for post-doctoral research in mathematical biology and optimal control theory for a year. He is currently serving as an associate professor at Necmettin Erbakan University, Turkey. His research interests mainly focus on infectious disease $d y$ namics, fractional mathematical modeling, fractional theory and method, optimal control theory and bifurcation analysis. He has published more than 60 research papers in international esteemed journals and he is a reviewer for about seventy international repute journals as well as he is Editor-in-Chief of the "Mathematical Modelling and Numerical Simulation with Applications" journal.

(iD https://orcid.org/0000-0002-3966-6518

Chandrali Baishya has obtained her PhD degree in Mathematical Sciences from the University of Mysore, Karnataka, India in 2009. She has been working as Assistant Professor in Tumkur University, Karnataka, India since 2011. Her areas of research interest are numerical analysis, fractional differential equations and mathematical biology.

(1) https://orcid.org/0000-0002-9653-313X

An International Journal of Optimization and Control: Theories \& Applications (http://ijocta.balikesir.edu.tr)

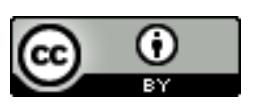

This work is licensed under a Creative Commons Attribution 4.0 International License. The authors retain ownership of the copyright for their article, but they allow anyone to download, reuse, reprint, modify, distribute, and/or copy articles in IJOCTA, so long as the original authors and source are credited. To see the complete license contents, please visit http://creativecommons.org/licenses/by/4.0/. 